\title{
MODELING SMART GRIDS AS COMPLEX SYSTEMS THROUGH THE IMPLEMENTATION OF INTELLIGENT HUBS
}

\author{
José González de Durana, Oscar Barambones \\ University College of Engineering, University of the Basque Country, Nieves Cano 12, 01006 Vitoria-Gasteiz, Spain \\ josemaria.gonzalezdedurana@ehu.es \\ Enrique Kremers, Pablo Viejo \\ European Institute for Energy Research, Karlsruhe Institute of Technology and EDF, \\ Emmy Noether Str. 11, 76131 Karlsruhe, Germany \\ kremers@eifer.org
}

Keywords: Electrical Grid, Hybrid Renewable Energy Systems, Energy Saving, Microgrid, Smart Meter, Intelligent Hub, Random Graph, Complex System, Complex Computer System, Scale Free Network, Agent Based Model.

\begin{abstract}
The electrical system is undergoing a profound change of state, which will lead to what is being called the smart grid. The necessity of a complex system approach to cope with ongoing changes is presented: combining a systemic approach based on complexity science with the classical views of electrical grids is important for an understanding the behavior of the future grid. Key issues like different layers and inter-layer devices, as well as subsystems are discussed and proposed as a base to create an agent-based system model to run simulations.
\end{abstract}

\section{The electrical grid as a Complex System}

The electrical grid as a whole can be considered as a complex system (more properly a Complex Computer System) whose aim is to assure a reliable power supply to all its consumers. Only regarding the grid from a multi-disciplinary point of view can help us understand the behavior of these systems. Despite conceptual advances in concrete fields like chaos theory or emergence in non-linear or self-organized systems, which were studied in the last decades, a unified theory of complexity does not yet exist.

Complex networks have been studied by several scientists. Erdös and Rényi (1959) suggested the modeling of networks as random graphs. In a random graph (Bollobás, 1998), the nodes are connected by a placing a random number of links among them. This leads to a Poisson distribution when considering the numbers of connections of the nodes, thus there are many nodes with a similar number of links.

Watts and Strogatz (1998) defined $\beta$ as the probability of rewiring an edge of a ring graph and called these networks small-world. Analyzing networks with values $0<\beta<1$, they found that these systems can be highly clustered, with a relatively homogenous topology, and have small characteristic path lengths.
However, the study of networks in the real world has shown that there are many examples where this is not true but they exhibit a common property: the number of links $k$ originating from a given node exhibits a power law distribution $P(k) \propto k^{-\gamma}$, i.e. few nodes having a large number of links. These networks are called scale-free and they are located in between the range of random and completely regular wired networks. Many systems in the real world such as neural networks, social networks and also the power grid, fulfill these properties.

Barabási and Albert (2002) mapped the topology of a portion of the World Wide Web and found that some nodes, which they called hubs, have many more connections than others and that the network as a whole exhibits a power-law distribution for the number of links connecting to a node. Using the BarabásiAlbert network model, Chassin and Posse (2005) analyzed the topologies of the North American electric grid to estimate their reliability and calculated the exponent of scale-free power law as being $\lambda=3.04$ for the U.S. eastern grid and $\lambda=3.09$ for the western one.

Considering all of the advancements in complexity science, in this paper we will show how an electricity grid can be represented through a model as a complex system that can be used for simulations. First, the smart grid will be presented and some key issues discussed. Then, the approach for modeling the grid is 
explained and in the last section the simulation model is presented.

\section{The smart grid}

The term smart grid as introduced by Amin and Wollenberg (2005), usually covers the entire spectrum of the electrical system, reaching from transportation over distribution up to the delivery. In common with earlier definitions, it contains two key elements: digital data processing and communication networks. Therefore, it can be said that what characterizes this intelligent grid is the existence of a flow of data and information, between the supplier company and the consumer, running in parallel with the energy flow (Singer, 2009).

With today's smart grid goals in mind, energy supply companies are in a transition process between our real electricity grid and the future smart grid, trying to improve the conventional network infrastructure, establishing the digital level (essence of the intelligent network) and also creating new business processes to carry out the capitalization and commercialization of the intelligent network.

The operation of the smart grid is far more complicated than the conventional power grid and in order to be operated, some special components like computers, sensors, remote controlled switching devices, as well as communication networks are necessary. For example, the current power grid is still not ready to admit microgrid connections. Connections made at present are experimental and almost always done manually, by taking care that a number of factors are fulfilled e.g. before realizing a connection.

Trying to model a microgrid, the network to which it will be connected should also be considered. Although a large amount of work in this area has been done, the main problem is that the current electricity grids are not yet adequately prepared for the transition to the smart grid. Therefore, in this article, the authors do not consider the inadequate existing grid, but instead focus on a hypothetical future network: the smart grid.

\subsection{The two layer model}

Concerning the upcoming challenges, especially facing the growing need of interaction of the different units of the smart grid, a two layer model is proposed (Kremers et al., 2010). These were identified as:

Physical layer: The first layer is the physical structure of the electrical grid itself, including all the power transmission lines. It includes the power

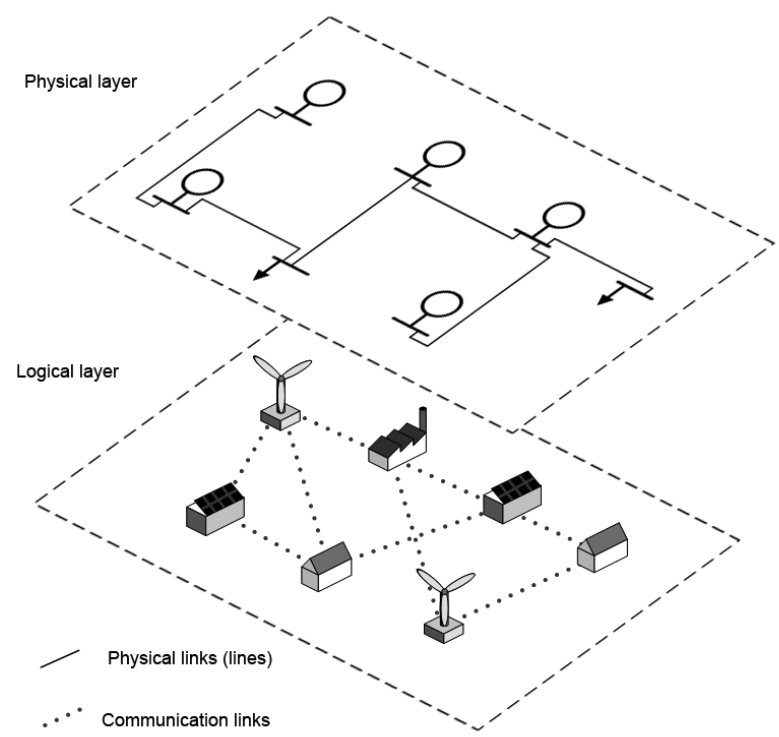

Figure 1: Different layers in an electrical microgrid

flows as well as all the electrical devices related to the correct operation of the grid.

Logical layer: This second layer, which represents the main part of the upcoming generation of electrical grids, is not yet present, in contrast to the physical layer. This layer includes all the information exchange that has to be arranged to control distributed generation (DG), dispatchable loads and other smart equipment in future grids. It has to be underlined that the communication paths do not have to be the same as the links in the first layer, although they could be exploited for that aim. An example of this is Power Line Communication (PLC).

The current electricity grid could be seen as part of the first layer, whereas the second layer is still the focus of vast research and development. It represents all the information and communication technology linked in some way to the grid and its operation. It implements a system that allows real-time communication between the elements of the grid. In the E-Energie project (2010), an Internet of Energy is suggested as an analogy to computer networks. This medium could itself serve as a communication platform. More examples of the implementation of the logical layer are described in Kremers et al. (2010) and could be PLC, existing communication networks, wireless technologies, etc.

In the following sections, some key role playing concepts of the smart grid will be exemplified and discussed. First of all, smart metering as a technol- 
ogy under deployment is presented. Afterwards, the concept of intelligent hub is introduced as a generic modeling approach for intelligent devices in the future grid. Finally, the microgrid as a sub-system of the smart grid is discussed.

\subsection{Smart Metering}

Traditionally, an energy meter measures only the consumption of the total energy during a specific time. This is used for billing the customer the total amount of energy he consumed. There is no way to obtain information on when the energy was consumed nor in what way. Smart meters are intended to provide more detailed information which will allow the supplier to adjust the pricing for consumption based on different parameters.

Electricity prices vary during a day or season, following the market offer-demand principle (especially with the introduction of renewable, non-dispatchable sources) or due to external factors such as temperature. Using a multiple tariff based system will allow for the reflection of these pricing changes to the final customer and thus entice him to make a more economical use of energy. These pricing signals shall help to reduce peak loads and sell more energy in offpeak periods, e.g. during the night.

The ESMA (European Smart Metering Alliance) defines a smart meter as an advanced meter with several functions, such as automatic data processing and transfer, automatic performing of measurements, which provides meaningful and up-to-date informations of consumption to the relevant actors and units of the energy system. Additionally, smart meters can provide support for measures to increase energy efficient consumption. Proof of the relevance of this device are the statements made by governments of different nations worldwide. For example, three of them have been chosen:

- Malta, where a pilot project is currently underway, with more than 5,000 smart counters being installed. The objective for 2012 is to have only smart meters in use.

- The United Kingdom, where in December 2009 the U.K. Department of Energy and Climate Change announced its intention to have smart meters in all homes by 2020 .

- The U.S. where, according to Edison's Institute for Electric Efficiency, many of the country's largest electricity distribution companies have plans to install millions of meters in the coming years, with deployments to be complete between 2012 and 2015.

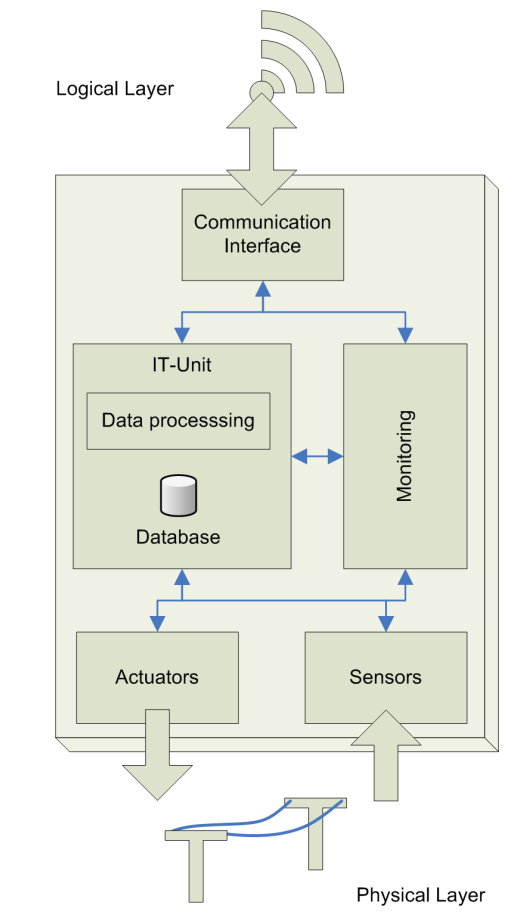

Figure 2: Architecture of an Intelligent Hub

However, in the authors' opinion, energy savings will only be achieved when the meters are reinforced by new devices and directives such as information displays, time-varying pricing, energy audits and, in particular, some form of automatic load control. The fact that companies such as Intel, Cisco or Google are developing hardware and software for this growing market, seems to confirm that idea.

\subsection{Intelligent Hubs as interaction between the layers}

Having described the smart grid properties, the question of how the assumed measures can be implemented to make the grid smarter is apparent. The approach taken in this study is to model some specially designed generic units called Intelligent Hubs that:

- implement the communication functions of the logical layer,

- monitor the physical grid,

- perform data processing and evaluation,

- can take actions on the physical grid,

- can act as a local decision unit, and

- handle any interactions between the logical and physical layer. 
The introduction of the intelligent hub arises from the idea that there are many different technologies and implementation possibilities for new infrastructure equipment, but no standard definition of these new intelligent units currently exists. For example, at household level a local load shedding module connected to a smart metering system could be implemented, or at a substation level new technologies that are be able to communicate with customers to send e.g. grid state signals, etc. are possible.

They all have in common that they share at least some of the characteristics of the intelligent hubs named above. This allows us to model a generic intelligent hub, which accomplishes with the specifications given and is able to simulate the behavior of these future equipment elements, even though a concrete implementation is not realized. It is important to underline that the intelligent hub is the link between the two layers, logical and physical, thus gathering information from both of them, being able to process it and actuating on the physical layer to perform changes. The acquisition of the data from the physical grid is performed by sensors, for example measuring units on the lines. The actuators are any kind of interaction with the power grid, such as demand control for example by direct (such as relays, operating on the line), or indirect means (dynamic demand reduction of the equipment).

The question of how far a smart meter can be seen as an intelligent hub or vice versa has to be analyzed further. There exist implementations of smart meters that seem to accomplish with some of the intelligent hub features (like load shedding functions), but in our opinion this already goes beyond the concept of metering. So, at least for modeling purposes, the smart meter will be seen as a part of the intelligent hub or an external unit linked to this, as the concept of the hub involves a much broader list of features, which can be summarized as the whole interaction between the two layers - even at different levels of the grid.

\subsection{Microgrids as smart grid subsystems}

A microgrid is a set of small energy generators arranged in order to supply energy for a community of users in close proximity. It is a combination of generation sources, loads and energy storage, interfaced through fast-acting power electronics. Emerging from the general trend of the introduction of Renewable Energy Sources (RES), microgrids will mostly include this type of generation, so they form part of the Hybrid Renewable Energy Systems (HRES). Microgrids represent a form of decentralization of electri-

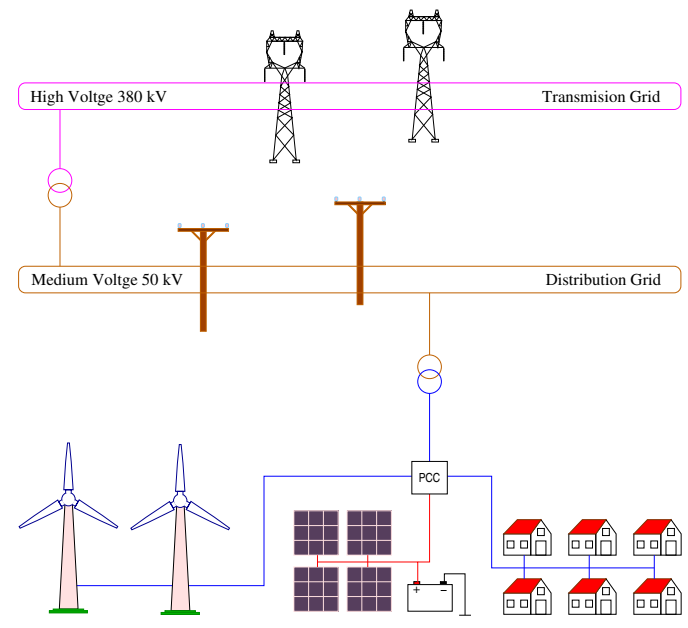

Figure 3: Integrated microgrid

cal networks. They comprise low- or medium-voltage distribution systems with distributed energy sources, storage devices and controllable loads.

During disturbances, the generation and corresponding loads can autonomously disconnect from the distribution system to isolate the load of the microgrid from the disturbance without damaging the integrity of the transmission grid. This mode is called islanding mode. From the point of view of the customer, it can be seen as a low voltage distribution service with additional features like an increase in local reliability, the improvement of voltage and power quality, the reduction of emissions, a decrease in the cost of energy supply, etc.

In Figure 3, a schematic drawing of an integrated microgrid can be seen, showing the Point of Common Coupling (PCC) and its electrical connections, but without representing the information channels. The authors have previously identified (Kremers et al., 2010) that electrical grids, as well as microgrids mostly satisfy the principal characteristics that distinguish them as true systems of systems, as defined by Maier (1998). The smart grid is constituted as a large complex system with operational and managerial independent elements (that are systems themselves), evolutionary development, emergent behavior and geographical distribution.

\section{A combined approach for smart grid modeling}

For simulation purposes, one should not pay attention to accessory components but instead focus on the essential parts. It may occur that modeling some im- 
portant elements is unnecessary for the operation, as for example some electronic components that while being essential for the actual operation they are trivial or nonexistent for simulation.

So in this article, we focus on a very important element to consider both the actual operation of the microgrid and the simulation: the smart meter. Assuming that there is an intelligent hub at each network bus, a smart meter at each load bus and there exists communication among the nodes, the resulting complex computer system can be used as a basis for smart grid models.

Agent-based modeling tools are able to recreate complex system behavior such as those described here, unexpected emergent behavior in these systems, internal and external events, communications within the system, etc. In particular, local effects of the single units comprising the system can be modeled and their effects can be analyzed at the system level.

The combination of several approaches allows the creation of models that might abstract some details from the single unit models, but all in all create a much more realistic representation at the system level. The inclusion of some communication among the devices is fundamental here. This combined approach is the one followed in this work, as in the authors' opinion it is very advantageous when modeling future electrical systems.

Geographic network localization, distribution of processing and databases, interaction with humans, and unpredictability of system reactions to unexpected external events are also present in this kind of network.

\section{The simulation model}

A combined approach model has been developed using Anylogic (XJTek, 2010), in which the grid nodes are represented by agents in the model, and each agent is provided of respective subsystem models (System Dynamics (SD), Discrete Events (DE), etc.). This kind of modeling has been chosen to allow for events such as the sudden elimination of one (or more) nodes, or connections between nodes, to be possible during the simulation, thus providing the possibility of a dynamic simulation of the electrical grid, including events such as failures, disasters and terrorist attacks.

The two-layer structure defined for the smart grid will be a key element due to its representation in the model. Apart from the agent-based approach, two other modeling paradigms are used: the SD paradigm for the physical layer and the DE paradigm for the logical layer.

The model is completely open, so it can be used to address a number of issues of design and computation that arise in such networks. Some of them coud be:

- Real grid, smart grid and microgrid simulation

- Grid and microgrid architecture design

- Centralized and decentralized control design

- Load connection and disconnection

- Microgrid connection and islanding modes

- Branch or node (e.g. substation) deleting

- Energy savings strategies

The approach presented here is intended to result in a suitable electrical microgrid model. It is a continuation of the authors' previous studies in this area (González de Durana et al., 2009; González de Durana and Barambones, 2009). In these works, the mesh method was used to obtain voltages at the mesh nodes and currents through the branches, assuming the voltages given by the generators are known. In a further study, however, a new power flow method has been created (Kremers et al., 2010). This new method was implemented using an combined approach in which agents represent the buses of the grid.

\section{Conclusion and outlook}

A view of energy systems from the complex systems approach has been given, underlining the importance of viewing the energy system as such, especially for its future development. Modeling the energy system at system level is crucial to help us understand the interactions of the single units, and be able to observe system phenomena such as emergence and the behavior of the system as a whole. The requirement of the introduction of new perceptions of the energy system was shown with the proposition of the two layer model to represent the smart grid. Further, a device abstraction was done for modeling generic devices called intelligent hubs that are able to interact with this new environment.

An agent-based model for the simulation of microgrids is being implemented using AnyLogic. The model offers a clear two-layer structure, which allows for the representation of both physical and logical interactions between the elements. The logical layer offers a robust base to implement agent communication in real time. The physical layer provides the technical results of the power flow calculation integrated into the model. This allows real-time simulations of the grid to be computed, which can provide valuable information prior to the implementation of the real grid. 
The first models of the intelligent hub have been developed and are currently being tested.

The model is mainly intended to design and test microgrids and can be used as a tool for the design, development and demonstration of control strategies, especially centralised supervisor control and decentralised load-dispatch control, the design and demonstration of microgrid operation strategies, the design and testing of microgrid communication buses and optimal microgrid design.

\section{ACKNOWLEDGEMENTS}

This work was made possible through cooperation between the University College of Engineering in Vitoria and EIFER in Karlsruhe. The authors are very grateful to the Basque Government for the support of this work through the project S-PE09UN12 and to the UPV/EHU for its support through the project GUI07/08.

\section{REFERENCES}

Amin, S. and Wollenberg, B. F. (2005). Toward a smart grid: power delivery for the 21 st century. Power and Energy Magazine, IEEE, 3(5):34-41.

Barabási, A. L. (2003). Linked: How everything is connected to everything else and what it means. Penguin Group New York.

Barabási, A. L. (2007). The architecture of complexity. Control Systems Magazine, IEEE, 27(4):33-42. 02721708.

Barabási, A. L. and Albert, R. (2002). Statistical mechanics of complex networks. Reviews of Modern Physics, 74.

Bollobás, B. (1998). Modern graph theory. Springer Verlag.

Chassin, D. P. and Posse, C. (2005). Evaluating north american electric grid reliability using the barabási-albert network model. Physica A: Statistical Mechanics and its Applications, 355(2-4):667-677.

Erdös, P. and Rényi, A. (1959). On random graphs, i. Publicationes Mathematicae (Debrecen), 6:290-297.

European Commission. (2006). European smartgrids technology platform: Visions and strategy for europe's electricity networks of the future.

E-Energie Projekt. (2010) Bundesministerium fuer Wirtschaft und Technologie. http://www.e-energie. de
González de Durana, J., Barambones, O., Kremers, E., and Viejo, P. (2009). Complete agent based simulation of mini-grids. In The Ninth IASTED European Conference on Power and Engineering Systems, EuroPES 2009, volume 681, pages 046-188, Palma de Mallorca, Spain. Acta Press.

González de Durana, J. M. and Barambones, O. (2009). Object oriented simulation of hybrid renewable energy systems focused on supervisor control. In IEEE Conference on Emerging Technologies and Factory Automation, 2009, ETFA 2009, pages 1-8.

Hatziargyriou, N., Asano, H., Iravani, R., and Marnay, C. (2007). Microgrids - an overview of ongoing research, development, and demonstration projects. IEEE power and energy magazine, (july/august 2007): 17.

Jiyuan, F. and Borlase, S. (2009). The evolution of distribution. Power and Energy Magazine, IEEE, 7(2):63-68. 1540-7977.

Karpov, Y. G., Ivanovski, R. I., Voropai, N. I., and Popov, D. B. (2005). Hierarchical modeling of electric power system expansion by anylogic simulation software. In Power Tech, 2005 IEEE Russia, pages 1-5.

Kremers, E., Lewald, N., Barambones, O., and González de Durana, J. (2009). An agent-based multi-scale wind generation model. In The Ninth IASTED European Conference on Power and Engineering Systems, EuroPES 2009, volume 681, pages 064-166, Palma de Mallorca, Spain. Acta Press.

Kremers, E., Viejo, P., González de Durana, J. M., and Barambones, O. (2010). A complex systems modelling approach for decentralized simulation of electrical microgrids. In 15th IEEE International Conference on Engineering of Complex Computer Systems, page 8, Oxford.

Maier, M. W. (1998). Architecting principles for systemsof-systems. Systems Engineering, 1(4):267-284.

Singer, J. (2009). Enabling tomorrow's electricity system report of the Ontario smart grid forum.

Valov, B. and Heier, S. (2006). Software for analysis of integration possibility of renewable energy units into electrical networks. In The 5th International Conference Electric Power Quality and Supply Reliability, Viimsi, Estonia. Proceedings Tallin University of Technology pp 173177.

Watts, D. J. and Strogatz, S. H. (1998). Collective dynamics of 'small-world' networks. Nature, 393(6684):440-442.

XJ Technologies website. (2010). Anylogic. http: //www . xjtek.com 